\title{
Influência do turno de rega no crescimento e produção do tomateiro no verão em Seropédica
}

\author{
José A Monte; Adilson de S Pacheco; Daniel F de Carvalho; Carlos Pimentel \\ UFRRJ, BR 465 km 07, 23890-000 Seropédica-RJ; jam1agr@yahoo.com.br; pacheco@fca.unesp.br; carvalho@ufrrj.br; \\ greenman@amcham.com.br
}

\section{RESUMO}

Com o objetivo de analisar a influência do turno de rega na cultura do tomateiro para mesa (híbrido Débora tipo longa vida), realizou-se um experimento de campo, no delineamento em faixas, com quatro tratamentos e quatro repetições, de 21/09/05 a 31/01/06. Foram avaliados ,o turno de rega diário (TR0); turno de rega alternado de um dia (TR1); turno de rega alternado de dois dias (TR2); e turno de rega alternado de três dias (TR3). Para todos os tratamentos foi reposto $100 \%$ da ETc. Durante o experimento, foram realizadas seis coletas para determinação da biomassa e da área foliar visando o estudo de análise do crescimento das plantas, sendo ajustadas equações para estimativa dos índices fisiológicos de crescimento da cultura. Foi possível constatar que o pico máximo de crescimento ocorreu entre 80 e 90 dias após o transplante, não sendo possível no entanto, diferenciar um tratamento que proporcionasse melhor crescimento. Os resultados obtidos indicaram que o turno de rega de três dias reduziu o número de frutos grandes ( $>60 \mathrm{~mm})$ e aumentou o número de frutos miúdos (40 a $50 \mathrm{~mm}$ ). Contudo, o turno de rega de dois dias proporcionou produção de tomate igual ou superior ao de turnos mais frequentes e com maior eficiência de uso de água. Para as condições de primavera-verão de 2005-2006 em Seropédica-RJ, o turno de rega alternado de dois dias mostrou-se mais favorável para recomendação aos agricultores.

Palavras-chave: Lycopersicon esculentum, área foliar, índice fisiológico, acúmulo de biomassa, qualidade de fruto.

\begin{abstract}
Influence of the irrigation schedule on growth and production of tomato during summer

The influence of the irrigation schedule was evaluated on the tomato culture, Debora hybrid for salad, in a field experiment, in a split plot design with four treatments and four replications, conducted from September, $21^{\text {st }}, 2005$ to January, $31^{\text {st }}, 2006$. We evaluated the irrigation every day (TR0); irrigation every two days (TR1); irrigation every three days (TR2); irrigation every four days (TR3). 100\% of the ETc was applied in all treatments. During the essay, six samplings of plants were performed to determine the plant weight and leaf area to adjust equations for the estimation of the growth parameters. The maximum growth was obtained between 80 and 90 days after transplanting, but in these experimental conditions the growth analysis did not discriminate the treatments. The irrigation schedule of three days caused a reduction in the number of big fruits $(>60 \mathrm{~mm})$ and increase in the number of small fruits $(40-50 \mathrm{~mm})$. However, the irrigation frequency of two days showed the same or higher fruit quality of the smaller intervals, but with improved water use efficiency. For the spring-summer of 2005-2006 conditions, at Seropédica, Rio de Janeiro State, Brazil, the irrigation schedule of two days was the best to be recommended for the farmer.
\end{abstract}

Keywords: Lycopersicon esculentum, leaf area, physiologic index, biomass accumulation, fruit quality.

\section{(Recebido para publicação em 11 de fevereiro de 2008; aceito em 27 de março de 2009)}

\section{(Received in February 11, 2008; accepted in March 27, 2009)}

$\mathrm{O}$ tomateiro (Lycopersicon esculentum Mill) é a segunda hortaliça mais cultivada no mundo, sendo sua produção superada apenas pela batata (Filgueira, 2003). A produção mundial de tomate alcançou cerca de 100 milhões de toneladas em 2003, sendo que o Brasil ocupa a oitava posição no ranking mundial, com aproximadamente $3 \%$ da produção (Cançado Junior, 2003). O elevado consumo se deve, principalmente, às suas qualidades organolépticas e a sua versatilidade culinária.

No estado do Rio de Janeiro, o cultivo do tomateiro é praticado com maior frequência durante o inverno, quando são observadas temperaturas mais amenas e favoráveis ao cultivo (Filgueira,
2003). Em contrapartida, no período de verão são verificadas menores quantidades de tomate ofertadas no mercado, motivo pelo qual maiores preços deste produto são esperados neste período. Mesmo em condições climáticas adversas, muitos produtores arriscam o cultivo no verão, em busca de maior lucratividade. Embora seja uma estação chuvosa, é muito comum a ocorrência de períodos de estiagem, denominados veranicos, justificando o uso da irrigação a fim de se obter o maior número de frutos com padrão comercial adequado (Bernardo et al., 2006).

$\mathrm{O}$ valor comercial do tomate de mesa é definido pelas características e qualidade presente no fruto (Ferreira et al., 2004), sendo que o tomateiro requer ade- quadas lâminas de água em todo o seu ciclo. De acordo com Filgueira (2003), as condições de umidade no solo, geralmente influenciam o rendimento da cultura, em função de seu efeito no número de flores por planta, na porcentagem de pegamento dos frutos e no tamanho dos frutos, pois o tomateiro é classificado como planta muito sensível ao estresse hídrico. Lopes et al. (2005) comentam que as variações de umidade do solo podem acarretar em queda de flores e desbalanceamento de cálcio, causando a podridão apical. Na maturação, as variações de umidade do solo podem causar rachaduras nos frutos, reduzindo a produção comercial. Entre os estádios fenológicos da cultura, o início da frutificação e o início do desenvolvimen- 
to dos frutos apresentam maior sensibilidade à deficiência de água no solo.

A adoção de turno de rega variável para fins de manejo de água envolve custos com a aquisição de sensores de umidade e uso de mão-de-obra para a leitura dos mesmos. Segundo Prieto et al. (1999), tais características desagradam à maioria dos agricultores, que preferem adotar turnos de rega fixos, permitindo melhor planejamento das práticas culturais e de outras atividades na propriedade. No entanto, recomendações para o manejo de água com base em turno de rega, devem ser determinadas para condições específicas, pois são muito afetadas pelas condições do clima e do solo.

A análise de crescimento tem utilização prática nos campos da agronomia e ecologia, pois é aplicada nos estudos de adaptabilidade ecológica das plantas. Por exemplo, a análise de crescimento é utilizada na adequação de uma determinada cultura a um determinado local (Rebouças et al., 1989), na avaliação de práticas agronômicas como manejo e tratos culturais (Andrade et al., 2005) e na seleção de genótipos por meio da capacidade produtiva (Guimarães et al., 2008). Também, o efeito da deficiência hídrica na taxa de acúmulo de matéria seca e de expansão foliar pode ser estudado utilizando-se a análise de crescimento (Gomes et al., 2000). De posse desses dados, torna-se mais efetiva a indicação de cultivares para condições climáticas e de manejo específicos.

Desenvolveu-se este trabalho com o objetivo de avaliar a influência do turno de rega na acumulação de massa seca e na produção comercial dos frutos de um híbrido de tomate para mesa, em Seropédica-RJ.

\section{MATERIAL E MÉTODOS}

O ensaio foi instalado em campo experimental da UFRRJ, de 21/09/05 a 31/ 01/06, em um solo classificado como Argissolo Vermelho-Amarelo, com $64,9 \%$ de areia, $7,6 \%$ de silte e $27,5 \%$ de argila nos primeiros $0,2 \mathrm{~m}$ de profundidade. A análise química do solo apresentou as características: $16 \mathrm{mg} \mathrm{L}^{-1} \mathrm{de}$ P, $0,2 \mathrm{cmol}_{\mathrm{c}} \mathrm{dm}^{-3} \mathrm{de}$ de $\mathrm{K}, 2,4 \mathrm{cmol}_{\mathrm{c}} \mathrm{dm}^{-3} \mathrm{de}$ $\mathrm{Ca}, 2,3 \mathrm{cmol}_{\mathrm{c}} \mathrm{dm}^{-3} \mathrm{de} \mathrm{Mg}, 0 \mathrm{cmol}_{\mathrm{c}} \mathrm{dm}^{-3} \mathrm{de}$ $\mathrm{Al}, \mathrm{pH}$ (em água) $5,1, \mathrm{e} 60 \%$ de saturação por base. O preparo do solo consistiu de aração e gradagem, quando foram aplicados $800 \mathrm{~kg} \mathrm{ha}^{-1}$ de calcário dolomítico. A adubação mineral consistiu na aplicação de $60 \mathrm{~kg} \mathrm{ha}^{-1} \mathrm{de} \mathrm{N}, 60 \mathrm{~kg} \mathrm{ha}^{-1} \mathrm{de} \mathrm{P}_{2} \mathrm{O}_{5}$ e 40 $\mathrm{kg} \mathrm{ha}^{-1}$ de $\mathrm{K}_{2} \mathrm{O}$ nas formas de uréia, superfosfato simples e cloreto de potássio, a lanço em superfície.

Cada parcela teve dimensões de 2,4 $\mathrm{m}$ de largura e 9,0 m de comprimento, tendo duas linhas, com 18 plantas em cada linha, para as coletas e avaliação da produção. Aos 35 dias após semeadura (DAS), foi realizado o transplante das mudas de tomateiro, híbrido Débora Plus, sendo utilizado um espaçamento entre linhas de $1,2 \mathrm{~m}$ e entre plantas de $0,5 \mathrm{~m}$. A cultura foi conduzida com uma haste por planta, sendo realizada a capação do ponteiro após a $6^{\circ}$ penca, com tutoramento vertical realizado por meio de fitilhos de plástico. O controle de plantas invasoras foi realizado por meio de capina manual.

A estimativa da lâmina de irrigação foi calculada utilizando a evapotranspiração de referência (ETo) gerado pelo Tanque Classe A, multiplicando a evaporação do Tanque Classe A por 0,78 (kp) e pelos valores do coeficiente de cultivo (kc) para conversão em (ETc). Os valores de kc utilizados foram os propostos por Doorenbos \& Kassan (1994). A irrigação foi realizada com tubos-gotejadores de polietileno com espaçamento entre emissores de $0,3 \mathrm{~m} \mathrm{e}$ vazão correspondente a $1,14 \mathrm{~L} \mathrm{~h}^{-1}$, sendo o seu manejo realizado com base nas leituras diárias de evaporação obtidas no tanque classe A.

O ensaio foi montado no delineamento em faixas, com quatro tratamentos e quatro repetições, em que os tratamentos foram frequências de irrigação, onde TR0 corresponde à reposição de água diária; TR1 corresponde ao turno de rega alternado de 1 dia; TR 2 corresponde ao turno de rega alternado de 2 dias; e o TR3 corresponde ao turno de rega alternado de 3 dias. Em todos os tratamentos, foi reposta $100 \%$ da evapotranspiração da cultura (ETc) e o cálculo do tempo de irrigação (Ti) foi realizado pela equação 1 :

$$
\text { Ti } \frac{(\text { ETo kc 0,12 60) }}{1,14}
$$

em que Ti= tempo de irrigação em minutos; $\mathrm{ETo}=$ evapotranspiração da cultura de referência, $\mathrm{mm} \mathrm{dia}^{-1} ; \mathrm{kC}=$ coeficiente de cultivo do tomateiro; $0,12=$ coeficiente referente à área $\left(\mathrm{em} \mathrm{m}^{2}\right)$ equivalente a um emissor; $60=$ se refere à conversão de hora para minutos; e 1,14= é a vazão de cada emissor em $\mathrm{L} \mathrm{h}^{-1}$.

Afim de se estudar o crescimento do tomateiro, foram realizadas seis coletas de material durante o experimento aos $55 ; 69 ; 90 ; 97 ; 125$ e 132 dias após o transplante, sendo retirada uma planta por parcela em cada avaliação. As plantas úteis, utilizadas para determinação da taxa de crescimento da cultura, foram obtidas previamente e aleatoriamente por coleta dentro de cada parcela. Em cada avaliação, as plantas coletadas foram levadas ao laboratório, onde realizou-se a separação em caules, folhas e frutos. Cada parte foi embalada adequadamente e levada à estufa de ventilação forçada, à temperatura de $80^{\circ} \mathrm{C}$, até atingir a massa seca constante. A área foliar foi estimada por meio de amostragem de 20 discos com área de $1,5393 \mathrm{~cm}^{2}$ de folha por planta, que foram secados e pesados separadamente, fazendo-se uma relação entre a massa seca do disco e sua área para ser aplicada à massa seca total das folhas e obter a área foliar total.

Foram utilizadas as equações matemáticas para ajuste dos dados, ao longo do tempo, do tipo exponencial polinomial do $2^{\circ}$ grau (equação 2 ). A partir dessas equações foram derivadas as taxas de crescimento da cultura (TCC) e de assimilação líquida (TCA), assumindo implicitamente que o crescimento da cultura é uma função do tempo (Pereira \& Arruda, 1987):

$$
\left.Y \quad e^{(a} \quad b x \quad c x^{2}\right)
$$

em que $\mathrm{Y}=$ valor da variável em estudo; $a, b$ e c= constantes de ajuste da regressão polinomial do $2^{\circ}$ grau; e $\mathrm{x}=$ dias após transplante (DAT).

Em seguida foi realizado o ajuste do modelo matemático e calculados os dados ajustados, sendo o coeficiente de determinação $\left(\mathrm{r}^{2}\right)$ o parâmetro utilizado como critério de seleção da função.

A taxa de crescimento da cultura (TCC, em $\mathrm{g} \mathrm{m}^{2}$ dia $^{-1}$ ) foi obtida pela diferença entre o acúmulo de matéria seca por área de solo, (entre coletas sucessivas), dividido pelo intervalo de tempo 


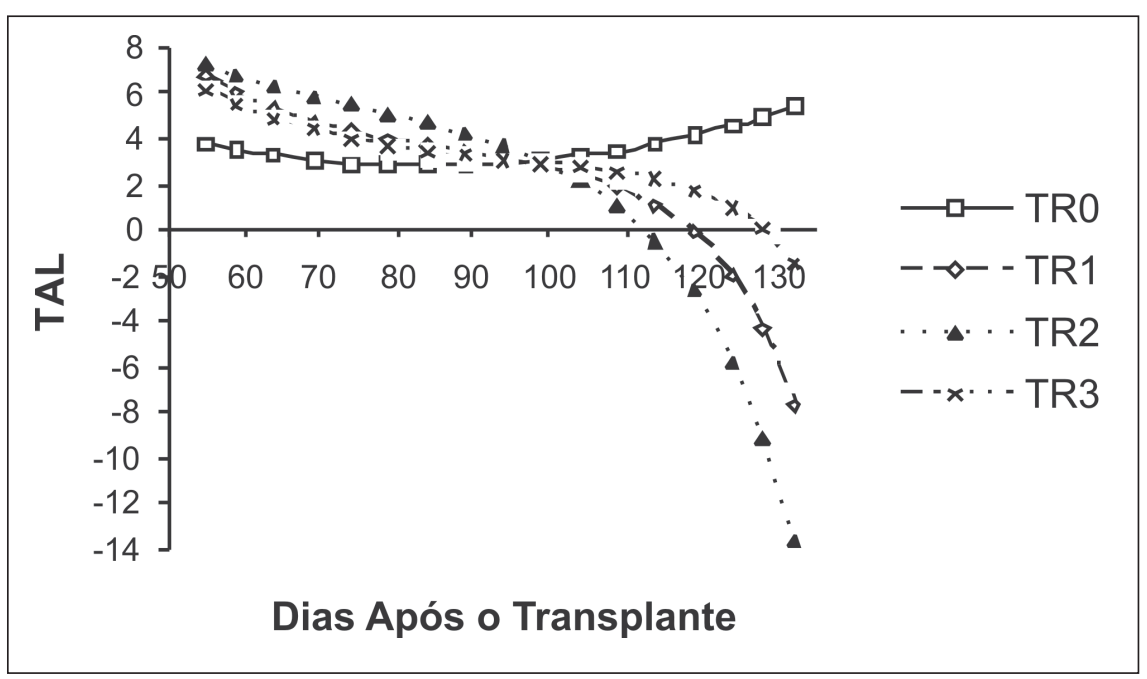

Figura 1. Taxa de Assimilação Líquida expressa em gramas de massa seca acumulada por área de folha em $\mathrm{m}^{2}$ por dia $\left(\mathrm{g} \mathrm{m}^{2} \mathrm{dia}^{-1}\right)$; valores instantâneos calculados dos quatros tratamentos; TR0 $=$ turno de rega diário, TR $1=$ turno de rega alternado de 1 dia, TR2 = turno de rega alternado de 2 dias e TR3= turno de rega alternado de 3 dias (net assimilation rate in grams of dry weight accumulated per leaf area in $\mathrm{m}^{2}$ per day $\left[\mathrm{g} \mathrm{m}^{2} \mathrm{dia}^{-1}\right]$; instantaneous values calculated for the four treatments; TR $0=$ irrigation every day, TR $1=$ irrigation every two days, TR2 $=$ irrigation every three days,TR3= irrigation every four days). Seropédica, UFRRJ, 2005-2006.

(equação 3), segundo Pereira \& Arruda (1987):

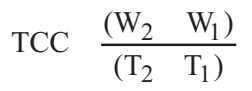

em que $\mathrm{W}=$ valor da fitomassa total por unidade de superfície cultivada (W $\mathrm{m}^{-2}$ ), resultante da soma da massa seca de caules, folhas e dos frutos, que foram obtidas ao longo das avaliações; $\mathrm{T}=$ dias após o transplante.

Para a determinação dos valores instantâneos da taxa de produção de matéria seca da cultura (TCC) foi empregada a derivada da equação, ajustada da curva de acúmulo de matéria seca por unidade de área de solo $\left(\mathrm{W} \mathrm{m}^{-2}\right)$, em relação ao tempo.

O índice de área foliar (IAF) foi obtido pela divisão da área foliar média de uma planta, em $\mathrm{m}^{2}$, pela área ocupada por uma planta $\mathrm{em}^{2}$, sendo ajustado interativamente a uma função exponencial polinomial do $2^{\circ}$ grau (equação 2). Para se obter a taxa de assimilação líquida (TAL, em $\mathrm{g} \mathrm{m}^{2} \mathrm{dia}^{-1}$ ) foi efetuado o cálculo utilizando os valores instantâneos da taxa de produção de matéria seca total, dividido pela área foliar, segundo as equações:

$$
\text { TAL } \frac{\left.(b 2 c x) e^{(a b x} c^{2}\right)}{e^{\left(a^{\prime} b^{\prime} x c^{\prime} x^{2}\right)}}
$$

$$
\mathrm{TCC}=\mathrm{TAL} * \mathrm{IAF}(5)
$$

em que $\mathrm{a}, \mathrm{b}$ e $\mathrm{c}=$ constantes de ajuste da regressão polinomial do $2^{\circ}$ grau; e $\mathrm{x}=$ dias após transplantio (DAT).

A quantificação da produção dos frutos do tomateiro foi efetuada a partir de valores obtidos da produção média de dez plantas a as classes de frutos foram estabelecidas conforme as normas da CEASA-MG (Ceasa, 2008).

A análise de variância foi efetuada no delineamento de parcelas subdivididas no tempo, sendo avaliados o efeito dos tratamentos e da interação entre os tratamentos e coletas. Também foi realizada a análise de regressão e o teste SNK a 5\% de significância, para comparação da produção dos frutos do tomateiro dentro dos tratamentos.

Os dados foram analisados através do programa de análise estatística SISVAR 5.0.

\section{RESULTADOS E DISCUSSÃO}

A evapotranpiração da cultura estimada durante o período experimental foi de 290,8 mm. Os valores médios das temperaturas máxima, mínima e média foram de 37,20 e $34^{\circ} \mathrm{C}$, respectivamente. As lâminas brutas de irrigação aplicada foram: no TR $0=338,25 \mathrm{~mm}$, no TR $1=$ $323,31 \mathrm{~mm}$, no TR $2=284,93 \mathrm{mmeno} \mathrm{TR} 3=$
287,00 mm. Durante o período experimental registrou-se uma precipitação acumulada de $600 \mathrm{~mm}$, colaborando, dessa forma, para maior homogeneização da umidade do solo e dificultando a diferenciação entre os tratamentos aplicados. Segundo Doorenbos \& Kassan (1994), as necessidades hídricas totais, após o transplante, para a cultura do tomateiro no campo com 90 a 120 dias (ETc) são de 400 a $600 \mathrm{~mm}$, que foram supridas, em parte, pela precipitação total. No entanto, as precipitações ocorreram desuniformemente distribuídas podendo ter ocasionado pequenos déficit hídrico entre precipitações consecutivas.

Segundo Alvarenga (2000), a irrigação excessiva durante o período de floração, tem provocado aumento na queda de flores e redução no estabelecimento de frutos. Neste estádio fenológico da cultura foram observadas precipitações frequentes, que causaram os efeitos descritos anteriormente. Segundo Sobral (1987), em temperaturas acima de $35^{\circ} \mathrm{C}$ ocorre a diminuição da fecundação das flores e queda de flores e de frutos. Temperaturas superiores a esta foram registradas durante vários dias ao longo do experimento.

Quanto ao crescimento das plantas, a TAL representa o balanço entre a massa produzida pela fotossíntese bruta e o que foi perdido através da respiração (Pereira \& Machado, 1987), ou seja, ela representa a variação da massa orgânica formada num dado momento, permitindo, dessa forma, estimar a fotossíntese líquida, indicando a eficiência do mecanismo fotossintético. Nos valores da TAL houve um padrão regular entre 55 e 110 DAT (Figura 1). A partir dos 110 DAT foi observada uma diferenciação da TAL entre os tratamentos, sendo que o TR2 apresentou a maior redução, seguido dos TR1 e TR3, havendo um leve acréscimo nos valores da TAL do TR0. O decréscimo da TAL no final do ciclo é desejável para que não haja crescimento vegetativo competindo com o crescimento de frutos, como ocorreu TR2, seguido do TR1 e TR3. Já no TR0 houve crescimento vegetativo no final do ciclo, provavelmente devido à máxima hidratação das plantas (Doorenbos \& Kassan, 1994) e, por estar no final, este desenvolvimen- 
Tabela 1. Avaliação da massa dos frutos expressa em kg por planta, e do número de frutos por planta (evaluation of fruit mass in kg per plant, and the number of fruits per plant). Seropédica, UFRRJ, 2005-2006.

\begin{tabular}{lcccccccccc}
\hline TRAT $^{1}$ & MT $^{2}$ & MG & MM & MP & MD & NT & NG & NM & NP & ND \\
\hline TR0 & $1,58 \mathrm{ab}^{3}$ & $0,17 \mathrm{a}$ & $0,71 \mathrm{ab}$ & $0,19 \mathrm{~b}$ & $0,48 \mathrm{a}$ & $20,0 \mathrm{~b}$ & $1,4 \mathrm{a}$ & $7,9 \mathrm{ab}$ & $3,0 \mathrm{~b}$ & $7,7 \mathrm{a}$ \\
TR1 & $1,30 \mathrm{bc}$ & $0,07 \mathrm{ab}$ & $0,63 \mathrm{ab}$ & $0,21 \mathrm{~b}$ & $0,37 \mathrm{a}$ & $17,3 \mathrm{~b}$ & $0,4 \mathrm{ab}$ & $7,5 \mathrm{ab}$ & $3,7 \mathrm{~b}$ & $5,7 \mathrm{a}$ \\
TR2 & $1,79 \mathrm{a}$ & $0,19 \mathrm{a}$ & $0,85 \mathrm{a}$ & $0,19 \mathrm{~b}$ & $0,54 \mathrm{a}$ & $22,3 \mathrm{a}$ & $1,4 \mathrm{a}$ & $9,3 \mathrm{a}$ & $3,5 \mathrm{~b}$ & $8,1 \mathrm{a}$ \\
TR3 & $1,14 \mathrm{c}$ & $0,01 \mathrm{~b}$ & $0,48 \mathrm{~b}$ & $0,28 \mathrm{a}$ & $0,40 \mathrm{a}$ & $17,7 \mathrm{~b}$ & $0,1 \mathrm{~b}$ & $5,9 \mathrm{a}$ & $4,9 \mathrm{a}$ & $6,8 \mathrm{a}$ \\
\hline CV(\%) & 11,68 & 31,14 & 19,13 & 14,48 & 25,82 & 7,17 & 32,26 & 18,18 & 17,96 & 21,66 \\
\hline
\end{tabular}

${ }^{1}$ TR0, TR1, TR2, TR3= turno de rega diário, alternado de 1, 2 e 3 dias, respectivamente; ${ }^{2} \mathrm{MT}=$ massa de frutos total; $\mathrm{MG}=\mathrm{massa}$ de frutos grandes; $\mathrm{MM}=$ massa de frutos médios; $\mathrm{MP}=$ massa de frutos pequenos; $\mathrm{MD}=$ massa de frutos com defeitos; $\mathrm{NT}=$ número de frutos totais; $\mathrm{NG}=$ número de frutos grandes com diâmetro igual ou superior a $60 \mathrm{~mm}$; $\mathrm{NM}=$ número de frutos médios com diâmetro entre 50 a 60 mm; $\mathrm{NP}=$ número de frutos pequenos com diâmetro entre 40 a $50 \mathrm{~mm}$; ND= número de frutos com defeitos em função dos quatros tratamentos ${ }^{3}$ Médias seguidas de letras iguais são estatisticamente semelhantes entre si, ao nível de 5\% de probabilidade pelo Teste de Tukey ( ${ }^{1}$ TR0, TR1, TR2, TR3= irrigation every day, every two days, every three days, every four days, respectively; ${ }^{2} \mathrm{MT}=$ total fruits mass; $\mathrm{MG}=$ big fruit mass; $\mathrm{MM}=$ average fruits mass; $\mathrm{MP}=$ small fruits mass; $\mathrm{MD}=$ defectives fruits mass; $\mathrm{NT}=$ total fruit number; $\mathrm{NG}=\mathrm{big}$ fruits number, with a diameter higher than $60 \mathrm{~mm}$; NM= medium fruits number, with a diameter between 50 and $60 \mathrm{~mm}$; $\mathrm{NP}=$ small fruits number, with a diameter between 40 and $50 \mathrm{~mm}$; ND= defective fruits number on the four treatments; ${ }^{3}$ Mean values followed by the same letters are statistical equal at the level of $5 \%$ by the Tukey test).

to se torna desnecessário, uma vez que não haverá tempo dos fotoassimilados produzidos por estas folhas serem translocados para os frutos, visto que a produção de frutos neste tratamento não foi superior (Tabela 1). Como no caso do tomateiro o que interessa é a produção de frutos, nesta fase do ciclo, se a planta conseguir transferir o máximo de energia para a produção de frutos, em detrimento da parte vegetativa, maior será a produção de frutos de tomate.

Para o IAF é possível observar uma elevação do valor desde os 55 DAT até os 85-95 DAT, quando os valores máximos em todos os tratamentos foram verificados (Figura 2). A partir deste momento, foi observado um padrão de redução até os 132 DAT, com a senescência de folhas. O IAF aumenta durante o ciclo da cultura, atingindo um valor ótimo quando a TCC é máxima. Entretanto, grandes aumentos do IAF, podem causar decréscimo da produção de massa seca, devido ao autosombreamento das folhas.

Para todos os tratamentos, analisando-se a TCC foi observado o mesmo padrão de crescimento do tomateiro (Figura 3) com um crescimento lento no início, acelerando-se, em seguida, até atingir o valor máximo e reduzindo-se a partir desse ponto, pois à medida que a planta cresce e aumenta o seu número de folhas, ela possui maior capacidade de produzir fitomassa. Como a planta apresenta crescimento indeterminado, ela tenderia a continuar crescendo

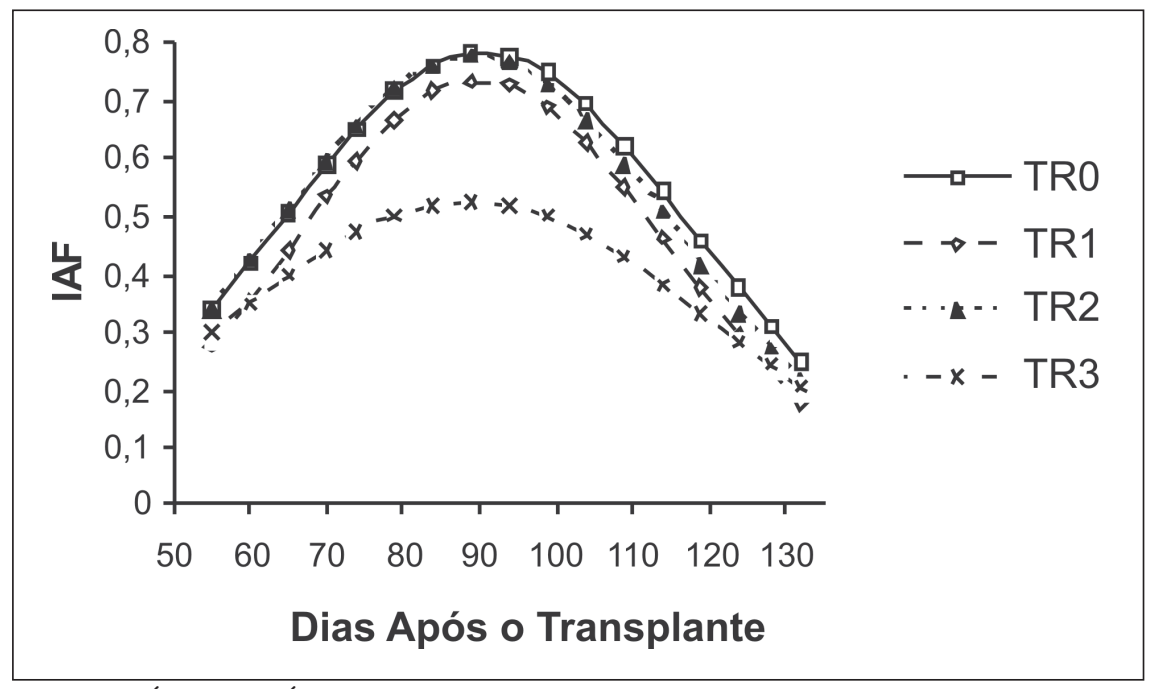

Figura 2. Índice de Área Foliar (IAF) para valores instantâneos calculados dos quatros tratamentos; TR $0=$ turno de rega diário, TR1 $=$ turno de rega alternado de 1 dia, TR2 $=$ turno de rega alternado de 2 dias e TR3= turno de rega alternado de 3 dias (leaf area index (IAF) for instantaneous values calculated for the four treatments; TR $0=$ irrigation every day, TR $1=$ irrigation every two days, TR2= irrigation every three days, TR3= irrigation every four days). Seropédica, UFRRJ, 2005-2006.

vegetativamente, competindo com o crescimento dos frutos. Porém, o crescimento final fica determinado pelo manejo da cultura, onde se elimina a gema apical, pois a partir de uma determinada altura não é mais conveniente manter o crescimento em altura, em virtude das dificuldades existentes.

As plantas com o turno de rega de dois dias tiveram um crescimento inicial mais acelerado e ocuparam o espaço máximo destinado a elas mais rapidamente (por volta dos 80 DAT). As plantas dos demais tratamentos atingiram a
TCC máxima num tempo maior, com valores inferiores (Figura 3). No entanto o decréscimo da TCC observado a partir dos 80 DAT, para o turno de rega de dois dias, e a partir dos 100 DAT, para os demais tratamentos, foi ocasionado pela senescência das folhas das plantas.

As taxas do IAF e da TCC do turno de rega de três dias apresentaram tendência em serem menores em relação às taxas dos demais tratamentos. Isso se deve provavelmente ao menor número de irrigações realizadas, em virtude dos valores de precipitações registrados no 


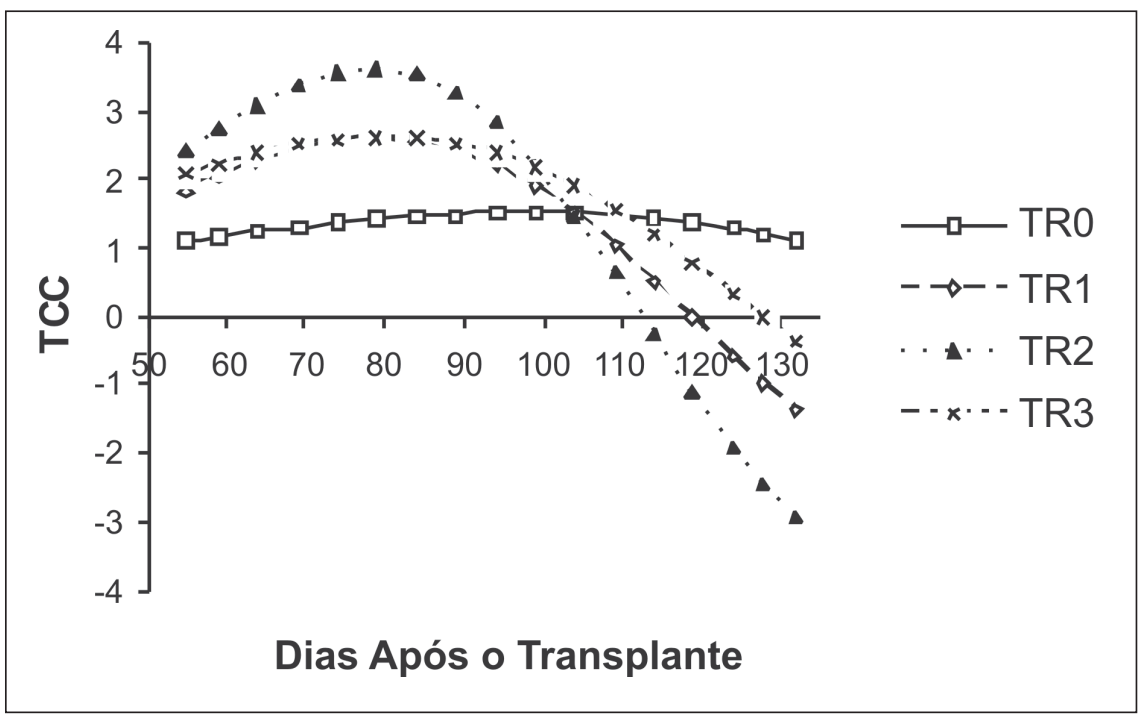

Figura 3. Taxa de Crescimento da Cultura, expressa em gramas de massa seca por área de solo em $\mathrm{m}^{2}$ por dia $\left(\mathrm{g} \mathrm{m}^{2} \mathrm{dia}^{-1}\right)$, para valores instantâneos calculados dos quatros tratamentos; $\mathrm{TR} 0=$ turno de rega diário, TR $1=$ turno de rega alternado de 1 dia, TR2 $=$ turno de rega alternado de 2 dias e TR $3=$ turno de rega alternado de 3 dias (crop growth rate, in grams of dry weight per soil area in $\mathrm{m}^{2}$ per day $\left(\mathrm{g} \mathrm{m}^{2} \mathrm{dia}^{-1}\right)$, for instantaneous values calculated for the four treatments; TR $0=$ irrigation every day, TR $1=$ irrigation every two days, TR $2=$ irrigation every three days, TR3= irrigation every four days). Seropédica, UFRRJ, 2005-2006.

período, pois na medida em que haveria necessidades de irrigações entre precipitações, as irrigações dos tratamentos com menor turno de rega iam sendo efetuadas, e durante o intervalo entre precipitações, estas irrigações foram repetidas mais vezes em comparação àquelas com um turno de rega maior. Neste caso, os tratamentos com menor turno de rega foram beneficiados com uma menor variação na umidade do solo, embora todos os tratamentos, teoricamente, ao final do ciclo devessem receber o mesmo volume de água, o que contribui para a tendência de menores taxas de IAF e TCC do turno de rega de três dias.

No geral, para os resultados da avaliação de massa de frutos e número de frutos por planta, em todos os tratamentos, observou-se maior número de frutos médios, com poucos frutos grandes e pequenos (Tabela 1). A produção de frutos pequenos do TR3 foi maior que nos outros tratamentos, tanto para massa quanto para números de frutos. Segundo Harmanto et al. (2004), a reposição de água com $75 \%$ da ETc, estimada pela equação de Penman-Monteith, mostrou melhor resultado em tomateiro cultivado em casa de vegetação. Resultados semelhantes foram obtidos por Dalvi et al. (1998), também em casa de vegetação, onde a irrigação por gotejamento programada a 79\% de ETc resultou em rendimento máximo para o tomateiro. Dessa forma, verifica-se que, para maior economia no gasto de água para a produção do tomateiro, não há necessidade de se repor $100 \%$ da ETc diariamente, como no TR0, e os turnos de rega menos freqüentes resultam na mesma produtividade (Tabela 1).

A alta umidade do solo, a partir de certo valor, pode reduzir a produtividade de frutos de qualidade no tomateiro (Harmanto et al., 2004). No presente estudo, nota-se que a irrigação diária, mantendo alta umidade do solo, não favoreceu ao aumento da produção de massa de frutos, ficando o tratamento com turno de rega de dois dias com produção equivalente ao turno de rega diário (Tabela 1), conforme os resultados de Marouelli \& Silva (2005). Já o turno de rega de três dias, que causa uma maior oscilação de umidade durante o cultivo, culminou com maior produção de frutos menores. Portanto, em termos econômicos, o turno de rega de 2 dias apresentou produção equivalente ao tratamento com turno de rega diário, e com maior eficiência na produção de frutos de tomate, sendo mais econômico e interessante para o agricultor, pois além de gastar menos água e energia, permite plane- jar outras atividades da propriedade com antecedência exigindo menor mão-deobra para o manejo de irrigação.

\section{AGRADECIMENTOS}

À Coordenação de Aperfeiçoamento de Pessoal de Nível Superior CAPES, pela concessão de bolsa de mestrado ao primeiro autor.

\section{REFERÊNCIAS}

ALVARENGA MAR. 2000. Cultura do tomateiro. Lavras: UFLA. 91p. (Textos Acadêmicos 2)

ANDRADE AC; FONSECA DM; LOPES RS; NASCIMENTO JUNIOR D; CECON PR; QUEIROZ DS; PEREIRA DH; REIS ST. 2005. Análise de crescimento do capimelefante 'napier' adubado e irrigado. Ciência e Agrotecnologia 29: 415-423.

BERNARDO S; MANTOVANI E; SOARES AA. 2006. Manual de irrigação. Viçosa: UFV. 625p.

CANÇADO JUNIOR FL; CAMARGO FILHO, WP; ESTANISLAU MLL; PAIVA BM; MAZZEI AR; ALVES HS. 2003. Aspectos econômicos da produção e comercialização do tomate para mesa. Informe Agropecuário 24: 7-18.

CEASA Minas Gerais, 2008. Disponível em: ht tp://www.ceasaminas.com.br/ agroqualidade/tomate.asp. Acesso em outubro de 2008 .

DALVI VB; TIWARI KN; PAWADE MN; PHIRKE PS. 1998. Response surface analysis of tomato production under microirrigation. Agricultural Water Management 12: 11-19.

DOORENBOS L; KASSAN AH. 1994. Efeito da água no rendimento das culturas. Campinas Grande: UFPR; 306p.

FERREIRA SMR; FREITAS RJS; LAZZARI EN. 2004. Padrão de identidade e qualidade do tomate (Lycopersicon esculentum Mill.) de mesa. Ciência Rural 34: 329-335.

FILGUEIRA FAR. 2003. Novo manual de olericultura; Agrotecnologia moderna na produção e comercialização de hortaliças. Viçosa: UFV. 412p.

GOMES AA; ARAÚJO AP; ROSSIELO ROP; PIMENTEL C. 2000. Acumulação de biomassa, características fisiológicas e rendimento de grãos em cultivares de feijoeiro irrigado e sob sequeiro. Pesquisa Agropecuária Brasileira 35: 1922-1937.

GUIMARÃES CM; STONE LF; NEVES PCF. 2008. Eficiência produtiva de cultivares de arroz com divergência fenotípica. Revista Brasileira de Engenharia Ambiental 12: 465-470.

HARMANTO VM, SALOKHE BMS; TANTAU HJ. 2004. Water requirement of drip irrigated tomatoes grown in greenhouse in tropical environment. Agricultural Water Management 71: 225-242. 
LOPES CA; ÁVILA AC; REIS A; INOUENAGATA A; QUEZADO-DUVAL AM; HENZ GP; CHARCHAR JM; BOITEUX LS; GIORDANO LB; MELO PCT. 2005. Doenças do Tomateiro. Brasília: Embrapa Hortaliças. $151 \mathrm{p}$.

MAROUELLI AW; SILVA WLC. 2005. Freqüência de irrigação por gotejamento durante o estádio vegetativo do tomateiro para processamento industrial. Pesquisa Agropecuária Brasileira 40: 661-666.
PEREIRA AR; ARRUDA HV. 1987. Ajuste prático de curvas na pesquisa biológica. Campinas: Fundação Cargil. 51p.

PEREIRA AR; MACHADO EC. 1987. Análise quantitativa do crescimento vegetal. Campinas: Instituto Agronômico. 33p. (Boletim Técnico, n.114)

PRIETO MH; LÓPEZ J; BALLESTEROS R. 1999. Influence of irrigation system and strategy of the agronomic and quality parameters of the processing tomatoes in Extremadura. Acta Horticulturae 487: 575-579.
REBOUÇAS MAA; FAÇANHA JGV; PEREIRA LGR; PRISCO JT. 1989. Crescimento e conteúdo de $\mathrm{N}, \mathrm{P}, \mathrm{K}$ e Na em três cultivares de algodão sob condições de estresse salino. Revista Brasileira de Fisiologia Vegetal 1: 79-85.

SOBRAL VS. 1987. Cultura do Tomate. Seropédica: EDUR-UFRRJ. 123p. 